\title{
Linear scaling electronic structure calculations and accurate sampling with noisy forces
}

\author{
Florian R. Krajewski and Michele Parrinello \\ Computational Science, Department of Chemistry and Applied Biosciences, \\ ETH Zurich, USI Campus, Via Giuseppe Buffi 13, CH-6900 Lugano, Switzerland
}

(Dated: June 21, 2018)

\begin{abstract}
Numerical simulations based on electronic structure calculations are finding ever growing applications in many areas of physics. A major limiting factor is however the cubic scaling of the algorithms used. Building on previous work [F. R. Krajewski and M. Parrinello, Phys.Rev. B 71, 233105 (2005)] we introduce a novel statistical method for evaluating the inter-atomic forces which scales linearly with system size and is applicable also to metals. The method is based on exact decomposition of the fermionic determinant and on a mapping onto a field theoretical expression. We solve exactly the problem of sampling the Boltzmann distribution with noisy forces. This novel approach can be used in such diverse fields as quantum chromodynamics, quantum Monte Carlo or colloidal physics.
\end{abstract}

PACS numbers: 71.15.-m, 31.15.-p

Keywords: electronic structure, linear scaling, tight binding, Langevin equation

Atomistic simulations in which the interactions are computed on the fly from electronic structure calculation play an important role in modern science and have proven their relevance in many fields. However their computational cost is a severe limitation. In particular simulating large systems has proven challenging due to the cubic dependence of the computation time on the number of electrons. This has long since been recognized 1, 2 and a number of algorithms have been suggested that in principle lead to linear scaling 3 , 4, 5, 6, 6, 8, 9, 10]. Most are based on the possibility in semiconductors or insulators of localizing the electronic orbitals. Linear scaling is then achieved by neglecting interactions between faraway atoms. This approach however suffers from poor convergence and leads to errors that are not easy to control. In metals the wavefunctions cannot be localized and only a handful of methods have been suggested [9, 10]. All in all it can be stated that in spite of considerable progress performing linear scaling ab-initio simulations is still very challenging.

Very recently we have proposed a new algorithm which scales linearly in all physical dimensions for semiconductors and metals alike 11. Here we reformulate the algorithm as a field theory. Sampling the resulting action is done stochastically. We show that, in spite of the statistical noise present in the evaluation of the forces, exact sampling can be performed. Our way of solving this problem is general and can also solve problems of similar nature that are encountered in quantum chromodynamics 12, 13], quantum Monte Carlo 14 and colloidal physics 15], where also the interaction can only be determined stochastically.

Let us start with the generic expression for the total energy in theories that can be formulated in an effective single particle form:

$$
E=2 \sum_{i=1}^{N} \epsilon_{i}+V_{r}
$$

The first term is the so-called band structure term given by the sum of the lowest $N$ doubly occupied eigenvalues $\epsilon_{i}$ of a Hamiltonian $\mathbf{H}$. For instance in density functional theory $\mathbf{H}$ is the Kohn and Sham Hamiltonian and $V_{r}$ corrects for double counting and accounts for the direct nuclear nuclear interaction, while in tight binding and other semi-empirical approaches $\mathbf{H}$ is a Hamiltonian which depends parametrically on the atomic positions and $V_{r}$ is a pairwise repulsive energy. In either case $V_{r}$ can be calculated in $\mathcal{O}(N)$ operations, while the calculation of the band structure term has an apparent $\mathcal{O}\left(N^{3}\right)$ complexity and has been the limiting factor that has so far prevented simulating very large systems.

Following ref. [16, 17] we write the band structure term as the low-temperature limit of the grand canonical potential for independent fermions:

$$
\Omega=-\frac{2}{\beta} \ln \operatorname{det}\left(1+e^{\beta(\mu-\mathbf{H})}\right) .
$$

In Eq. (2) $\mu$ is the electron chemical potential and it easy to see that $\lim _{\beta \rightarrow \infty} \Omega=2 \sum_{i=1}^{N} \epsilon_{i}-\mu N_{e}$, where $N_{e}=2 N$ is the total number of electrons. We now factorize the operator in Eq. (21) as:

$$
1+e^{-\beta(\mu-\mathbf{H})}=\prod_{l=1}^{P / 2}\left(\mathbf{M}_{l}^{\dagger} \mathbf{M}_{l}\right)
$$

where $P$ is an even integer and $\mathbf{M}_{l}=1+$ $e^{i \pi(2 l-1) / P} e^{\frac{\beta}{P}(\mu-\mathbf{H})}$. Here we depart from ref. 11] and since $\mathbf{M}_{l}^{\dagger} \mathbf{M}_{l}$ is a positive definite operator we can follow the practice well known in lattice gauge simulations 12 . 
of writing its inverse determinant as an integral over a field $\boldsymbol{\Phi}_{l}$ that has the dimension of the full Hilbert space in the form [18]:

$$
\operatorname{det}\left(\mathbf{M}_{l}^{\dagger} \mathbf{M}_{l}\right)^{-\frac{1}{2}}=\frac{\int \mathcal{D}\left[\mathbf{\Phi}_{l}\right] e^{-\boldsymbol{\Phi}_{l}^{\dagger} \mathbf{M}_{l}^{\dagger} \mathbf{M}_{l} \mathbf{\Phi}_{l}}}{\int \mathcal{D}\left[\mathbf{\Phi}_{l}\right] e^{-\mathbf{\Phi}_{l}^{\dagger} \mathbf{\Phi}_{l}}}
$$

Inserting the relation (3) into Eq. (2) after having used Eq. (4) we end up with the following expression for the grand canonical potential:

$$
\Omega=\frac{4}{\beta} \sum_{l=1}^{P / 2} \ln \int \mathcal{D}\left[\boldsymbol{\Phi}_{l}\right] e^{-\mathbf{\Phi}_{l}^{\dagger} \mathbf{M}_{l}^{\dagger} \mathbf{M}_{l} \boldsymbol{\Phi}_{l}}+\text { const. }
$$

which is the promised field theoretical formulation. The quantities of physical interest like energy or force can all be calculated as derivatives of $\Omega$ relative to an appropriate external parameter. For instance $N_{e}=-\frac{\partial}{\partial \mu} \Omega$, and assuming that $\beta^{-1}$ is so low that temperature effects on the electrons can be neglected $E^{\text {band }}=2 \sum_{i=1}^{N} \epsilon_{i}=$ $\frac{\partial}{\partial \beta}(\beta \Omega)+\mu N_{e}$ while the contribution to force on particle $I$ at position $\mathbf{R}_{I}$ coming from the band term is given by $\mathbf{F}_{I}^{\text {band }}=-\nabla_{\mathbf{R}_{I}} \Omega$. In taking the derivatives the constant in Eq. (5) vanishes and one is left with calculating expressions of the type:

$$
\frac{\partial \Omega}{\partial \lambda}=\frac{4}{\beta} \sum_{l=1}^{P / 2} \frac{\int \mathcal{D}\left[\boldsymbol{\Phi}_{l}\right]\left[\boldsymbol{\Phi}_{l}^{\dagger}\left(\frac{\partial \mathbf{M}_{l}^{\dagger}}{\partial \lambda} \mathbf{M}_{l}+\mathbf{M}_{l}^{\dagger} \frac{\partial \mathbf{M}_{l}}{\partial \lambda}\right) \boldsymbol{\Phi}_{l}\right] e^{-\boldsymbol{\Phi}_{l}^{\dagger} \mathbf{M}_{l}^{\dagger} \mathbf{M}_{l} \boldsymbol{\Phi}_{l}}}{\int \mathcal{D}\left[\boldsymbol{\Phi}_{l}\right] e^{-\boldsymbol{\Phi}_{l}^{\dagger} \mathbf{M}_{l}^{\dagger} \mathbf{M}_{l} \mathbf{\Phi}_{l}}}
$$

Thus all relevant properties can be evaluated by sampling the $P / 2$ distributions $e^{-\boldsymbol{\Phi}_{l}^{\dagger} \mathbf{M}_{l}^{\dagger} \mathbf{M}_{l} \boldsymbol{\Phi}_{l}}$.

So far no approximation has been made and no computational advantage has been gained either. In order to make further progress we must take advantage of the fact that in $\mathbf{M}_{l}$ the operator $e^{\frac{\beta}{P}(\mu-\mathbf{H})}$ appears and that $P$ can be taken to be sufficiently large for suitable approximations to the exponential operator to be accurate. In ref. [1] we used as basis set a grid in real space and a Trotter decomposition was the natural approximation to use. Here we will apply our method to a tight binding Hamiltonian and simply use a high-temperature expansion:

$$
\mathbf{M}_{l}=1+e^{\pi(2 l-1) / P}\left[1+\frac{\beta}{P}(\mu-\mathbf{H})\right]+\mathcal{O}\left(\left(\frac{\beta}{P}\right)^{2}\right)
$$

In such a manner the operator $\mathbf{M}_{l}$ has the same sparsity of $\mathbf{H}$, a fact which will eventually lead to linear scaling. We have also considered the possibility of using higher order expressions for the exponential operator in Eq. (7) 9]. This leads to a smaller $P$ value at the cost of a less sparse $M_{l}$. Since eventually we want to exploit parallelism as much as possible we have preferred to use the expression (7). Note that no assumption has been made on the energy spectrum or on the local character of the wavefunctions and therefore our method will be valid both for metals and non-metals. It simplifies the calculation of the properties of the system if in Eq. (6) we use the expression

$$
\frac{\partial \mathbf{M}_{l}}{\partial \lambda}=\frac{1}{2 P}\left\{\left(\mathbf{M}_{l}-1\right) \mathbf{O}_{\lambda}+\mathbf{O}_{\lambda}\left(\mathbf{M}_{l}-1\right)\right\}+\mathcal{O}\left(\frac{1}{P^{3}}\right) .
$$

which has an accuracy compatible with Eq. (7). In Eq. (8) $\mathbf{O}_{\lambda}$ can be $\beta,(\mu-\mathbf{H})$, or $-\beta \boldsymbol{\nabla}_{\mathbf{R}_{I}} \mathbf{H}$ for $\lambda=\mu$, $\beta$ or $\mathbf{R}_{I}$ respectively. A standard approach to sampling $e^{-\boldsymbol{\Phi}_{l}^{\dagger} \mathbf{M}_{l}^{\dagger} \mathbf{M}_{l} \boldsymbol{\Phi}_{l}}$ is to draw a sequence of normal distributed random numbers $\boldsymbol{\Psi}_{l}$ and compute $\boldsymbol{\Phi}_{l}$ solving the equations $\mathbf{M}_{l} \boldsymbol{\Phi}_{l}=\boldsymbol{\Psi}_{l}$. Since $\mathbf{M}_{l}$ is sparse, this equation can be solved in $\mathcal{O}(N)$ operations using for instance a biconjugated gradient method 19$]$. It can be easily shown that this approach is equivalent to the stochastic inversion method advocated in ref. [11]. Here we have decided instead to sample $e^{-\boldsymbol{\Phi}_{l}^{\dagger} \mathbf{M}_{l}^{\dagger} \mathbf{M}_{l} \boldsymbol{\Phi}_{l}}$ using a Langevin dynamics.

$$
m_{l} \ddot{\boldsymbol{\Phi}}_{l}=-\mathbf{M}_{l}^{\dagger} \mathbf{M}_{l} \boldsymbol{\Phi}_{l}-\gamma_{e} \dot{\boldsymbol{\Phi}}_{l}+\boldsymbol{\xi}_{l}
$$

where the components $\alpha$ of the white random noise vector $\xi_{l}$ obey the relation

$$
\left\langle\xi_{l}^{\alpha}(0) \xi_{l}^{\alpha}(t)\right\rangle=2 m_{l} \gamma_{e} \delta(t)
$$

In this way we circumvent the need to invert the matrix $\mathbf{M}_{l}$. This solves the problem that for a tight binding Hamiltonian as opposed to the Hamiltonian used in ref. [11] we were not able to find good preconditioners. Furthermore since different $\mathbf{M}_{l}$ 's have different eigenvalue spectra one can choose the $m_{l}$ so as to achieve the optimal sampling speed in each $l$ channel. This problem is particularly serious for metals where $\mathbf{M}_{l \approx \frac{P}{2}}$ can have eigenvalues close to zero, which leads to a much slower sampling speed 11]. Finally since we will use a Langevin sampling also for the ions it is pleasing to use the same sampling methodology for electronic and ionic degrees of freedom.

Inevitably the interatomic forces that are calculated by this procedure will be affected by a statistical error. This 
will prevent us from using these forces to perform energy conserving MD calculations. However we will show that sampling of the Boltzman distribution is still possible. Similarly to what was done for the electronic degrees of freedom we sample the ionic configurations with a Langevin equation:

$$
M \ddot{\mathbf{R}}_{I}=\mathbf{F}_{I}-\gamma_{I} \dot{\mathbf{R}}_{I}+\boldsymbol{\Xi}_{I}
$$

where the random force obeys the relations

$$
\left\langle\boldsymbol{\Xi}_{I}(0) \boldsymbol{\Xi}_{I}(t)\right\rangle=6 k_{\mathrm{B}} T M \gamma_{I} \delta(t)
$$

and

$$
\left\langle\mathbf{F}_{I}(0) \boldsymbol{\Xi}_{I}(t)\right\rangle=0
$$

From the electrons' Langevin dynamics we do not get the exact forces $\mathbf{F}_{\mathrm{I}}$ but an approximation $\mathbf{F}_{\mathrm{I}}^{L}=\mathbf{F}_{\mathrm{I}}+\boldsymbol{\Xi}_{\mathrm{I}}^{L}$ that is affected by a statistical error $\boldsymbol{\Xi}_{I}^{L}$ and therefore there is in principle no guarantee that correct Boltzman averages are obtained from the solution of Eq. (11). But let us assume, and we will show later in a realistic case that this assumption is indeed justified, that also $\boldsymbol{\Xi}_{I}^{L}$ is a white noise obeying

$$
\begin{aligned}
&\left\langle\boldsymbol{\Xi}_{I}^{L}(0) \boldsymbol{\Xi}_{I}^{L}(t)\right\rangle \cong 6 k_{\mathrm{B}} T M \gamma_{I}^{L} \delta(t) \text { and } \\
&\left\langle\mathbf{F}_{I}(0) \boldsymbol{\Xi}_{I}^{L}(t)\right\rangle \cong 0 .
\end{aligned}
$$

In this case the noise $\boldsymbol{\Xi}_{\mathrm{I}}^{L}$ simply adds to $\boldsymbol{\Xi}_{\mathrm{I}}$ and if we modify Eq. (11) so as to read

$$
M \ddot{\mathbf{R}}_{I}=\mathbf{F}_{I}-\left(\gamma_{I}+\gamma_{I}^{L}\right) \dot{\mathbf{R}}_{I}+\boldsymbol{\Xi}_{I}+\boldsymbol{\Xi}_{I}^{L}
$$

we recover a Langevin equation whose trajectories can still be used to obtain a Boltzman sampling.

At first sight it would appear that we are defeating our object since in general we know $\mathbf{F}_{I}+\boldsymbol{\Xi}_{I}^{L}$ but not each term individually. However we can determine $\gamma_{I}^{L}$ by varying it until the equipartition theorem $\left\langle\frac{1}{2} M \dot{\mathbf{R}}_{I}^{2}\right\rangle=\frac{3}{2} k_{\mathbf{B}} T$ is satisfied. With this choice the sampling will be correct and noisy forces can be used in the sampling. It is important to note that after the correct value for $\gamma_{I}^{L}$ is determined it has to be kept constant during the simulation. With this procedure one can exactly calculate static observables within the framework of Langevin dynamics without knowing the exact force. This is at variance with ref. 20] where explicit assumptions on the noise have to be made.

It remains now to show that the assumptions made above are correct and that the resulting scheme is accurate. As a test system we have chosen silicon in the diamond and in the liquid phase. We use the empirical tight binding interaction for silicon described in 21]. We checked that a value of $P=200$ is sufficient for the approximation of Eq. (7) to be valid. The Langevin dynamics parameters used were $\delta t_{e}=1, \gamma_{e} \delta t_{e}=1 / 20$ where $\delta t_{e}$
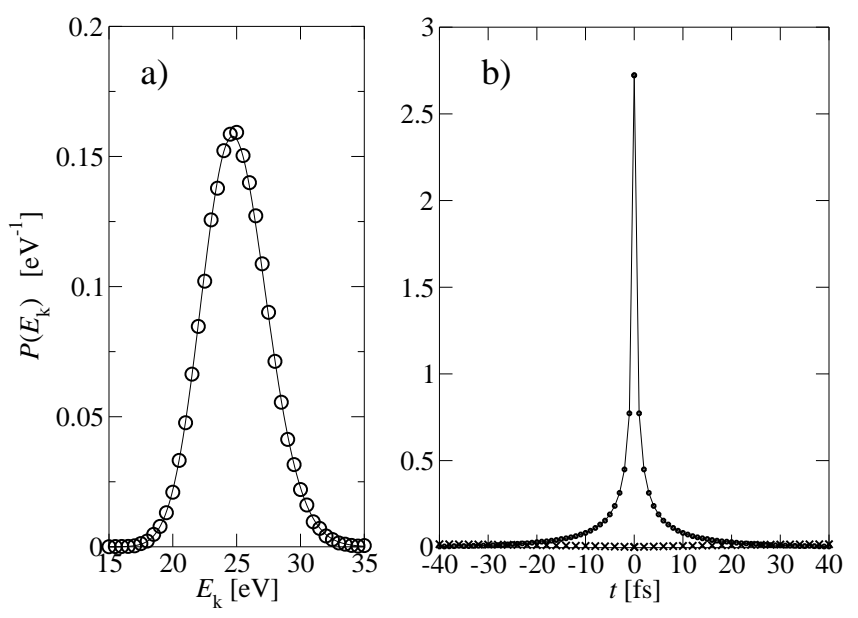

FIG. 1: a) Distribution of the kinetic energy for a simulation of liquid silicon with 64 atoms at $3000 \mathrm{~K}$ (circles). Maxwell distribution (line). b) Autocorrelation function of the noise $\left\langle\boldsymbol{\Xi}_{I}^{L}(0) \boldsymbol{\Xi}_{I}^{L}(t)\right\rangle /\left\langle\left(\mathbf{F}_{I}^{\text {band }}(0)\right)^{2}\right\rangle$ (line). Cross-correlation function of the noise and the exact force $\left\langle\mathbf{F}_{I}(0) \boldsymbol{\Xi}_{I}^{L}(t)\right\rangle /\left\langle\left(\mathbf{F}_{I}^{\text {band }}(0)\right)^{2}\right\rangle$ (crosses). The correlation functions are normalized to the exact ionic force stemming from the band energy. All results are calculated for a system 64 atoms of liquid silicon at $3000 \mathrm{~K}$.

is the discretized integration time step and the algorithm of Ricci and Ciccotti 22] has been used throughout. The masses $m_{l}$ are adjusted such that the average force fluctuations are $\left(\mathbf{M}_{l}^{\dagger} \mathbf{M}_{l} \boldsymbol{\Phi}_{l}\right)^{2} \frac{\delta t_{e}^{2}}{2 m_{l}}<0.025$. This adjustment of the masses is a crucial ingredient to obtain a good performance since if the masses were set to be equal for all values for $l$ the fastest time scales of the field $\Phi_{P / 2}$ would be more than one order of magnitude larger than that of $\Phi_{1}$. The integration time step for the ion dynamics is $\delta t=1 \mathrm{fs}$. After each ionic displacement we let the $\boldsymbol{\Phi}_{l}$ evolve under the action of the new $\mathbf{M}_{l}^{\dagger} \mathbf{M}_{l}$ until the distribution is equilibrated. The time needed for the equilibration is problem dependent and can be measured by looking at the correlation function $\left\langle\boldsymbol{\Phi}_{l}(0) \boldsymbol{\Phi}_{l}(t)\right\rangle$. In the present case we make the rather conservative choice of running the electronic Langevin equation for 100 time steps. The $\boldsymbol{\Phi}_{1}$ thus obtained are used to calculate the ionic forces for the next integration step. The chemical potential is continuously adjusted such that the number of electrons fluctuates around the desired value.

We first consider the case of $64 \mathrm{Si}$ atoms in a periodically repeated cell of length $10.86 \AA$ at a temperature of $3000 K$ where the system is metallic. Using the procedure described above we find that if we take $\gamma_{I}=\frac{1}{30} \mathrm{fs}^{-1}$ we need to add a correction due to the noise in the forces $\gamma_{I}^{L}=\frac{1}{379} \mathrm{fs}^{-1}$ which fixes the average kinetic energy of the particles at the desired value. In Fig. 11 a) it is also seen that not only is the average energy correct but also that its fluctuations follow Maxwell distribution. In this 


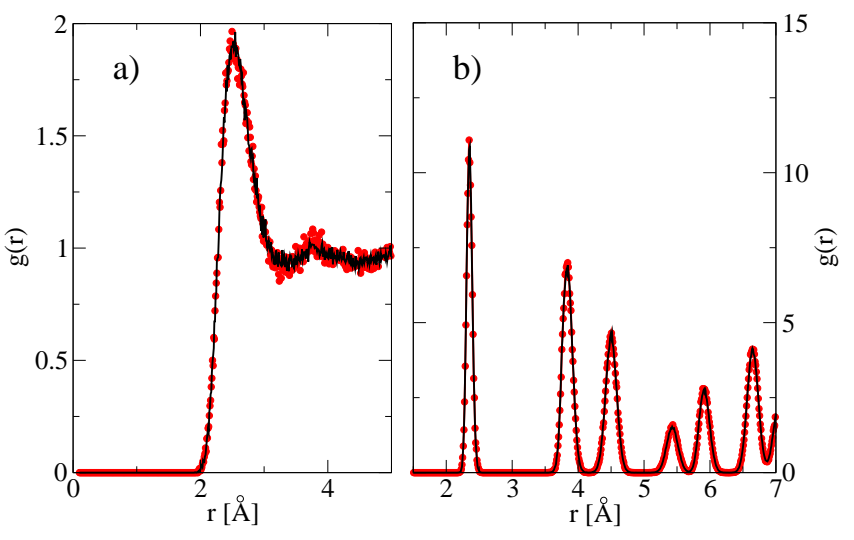

FIG. 2: (color) Pair correlation functions for liquid silicon $(3000 \mathrm{~K})$ (a) and crystalline silicon in the diamond phase $(300 \mathrm{~K})(\mathrm{b})$. The red dots show the results from our new method and the lines are calculated using standard diagonalisation of the tight binding Hamiltonian.

small system it is possible to calculate the correct forces on the ions and check the statistical properties of $\boldsymbol{\Xi}_{I}^{L}$. It is seen from Fig. 1 b) that Eq. (14) is satisfied to a very good approximation and that the correlation between the exact forces and the noise is minimal. Furthermore $\left\langle\boldsymbol{\Xi}_{I}^{L}(0) \boldsymbol{\Xi}_{I}^{L}(t)\right\rangle$ is strongly localized in time and its behavior is well fitted by two exponentials whose decay is much faster than the fastest ionic motion time scale. Therefore its net effect is similar to that of a delta function and in fact if we approximate $\left\langle\boldsymbol{\Xi}_{I}^{L}(0) \boldsymbol{\Xi}_{I}^{L}(t)\right\rangle$ as a delta function whose strength is given by its integral we find an estimate for $\gamma_{I}^{L} \approx \frac{1}{345} \mathrm{fs}^{-1}$ in good agreement with the empirical determination. Similar analysis conducted on semiconducting Si leads to a similar conclusion, namely that the effect of the noise in the calculation of the forces can be simply accounted for by adding the damping coefficient $\gamma_{I}^{L}$. In the solid phase we find $\gamma_{I}^{L}=\frac{1}{502} \mathrm{fs}^{-1}$ which is similar to the result for the liquid. Therefore we expect that, with some adjustment phase transitions can be studied with our method, at least in this case.

In Fig. 2 we compare the pair correlation functions $g(r)$ calculated with noisy forces and those evaluated with a standard approach. It is seen that the agreement is excellent and that the use of noisy forces does not degrade the quality of the simulation.

There are two issues that we would like to discuss briefly. One is the break-even point between standard calculations and the present linear scaling method. This is not easy to determine since it depends on the accuracy required, which in our case is related to the number $P$ used in the decomposition of the fermion determinant Eq. (3). For the case of Si we estimate on a single processor the crossing point to be at about 500 atoms. In other cases larger values of $P$ might be necessary, thus shifting the crossing point to larger systems. However our algorithm is trivially parallelizable and therefore we expect the situation to be much more favorable in terms of wall clock time on massive parallel platforms. Our code scales essentially linearly with $P$ while the diagonalizion codes, although highly developed, are not amenable to similarly efficient parallelization. For instance Shellman et al.23 have estimated that parallelization of a tight binding simulation on 1000 atoms loses efficiency when distributed over more than 8 processors. In addition we can gain even more if we organize our calculation hierarchically and distribute the most expensive part of the calculation, the matrix multiplication $\mathbf{M}_{l}^{\dagger} \mathbf{M}_{l} \boldsymbol{\Phi}_{l}$, to several processors.

Finally we stress once again that the interest in our work transcends the field of linear scaling algorithms and offers an alternative to other MC methods with noisy estimators 13,20 .

We thank A. Laio for his critical reading of the manuscript.

[1] W. Yang, Phys. Rev. Lett. 66, 1438 (1991).

[2] G. Galli and M. Parrinello, Phys. Rev. Lett. 69, 3547 (1992).

[3] S. Goedecker, Rev. Mod. Phys. 71, 1085 (1999).

[4] A. A. Mostofi, P. D. Haynes, C. Skylaris and M. C. Payne, J. Chem. Phys. 119, 8842 (2003).

[5] G. E. Scuseria, J. Phys. Chem. A 103, 4782 (1999).

[6] W. Kohn, Phys. Rev. Lett. 76, 3168 (1996).

[7] E. Hernandez and M. J. Gillan, Phys. Rev. B 51, 10157 (1995).

[8] X. P. Li, R. W. Nunes and D. Vanderbilt, Phys. Rev. B 47, 10891 (1993).

[9] S. Goedecker and L. Colombo, Phys. Rev. Lett. 73, 122 (1994).

[10] R. Baer and M. Head-Gordon, Phys. Rev. Lett. 79, 3962 (1997).

[11] F. R. Krajewski and M. Parrinello, Phys. Rev. B 71 , 233105 (2005). (2005).

[12] I. Montvay and G. Münster, Quantum Fields on a Lattice, Cambridge Monographs on Mathematical Physics (1994).

[13] G. Bhanot and A. D. Kennedy, Phys. Lett. 157B, 70 (1985).

[14] C. Pierleoni, D. M. Ceperley and M. Holzmann Phys. Rev. Lett. 93, 146402 (2004).

[15] F. Bitzer, T. Palberg, H. Löwen, R. Simon and P. Leiderer, Phys. Rev. E 50, 2821 (1994).

[16] A. Alavi and D. Frenkel, J. Chem. Phys. 97, 9249 (1992).

[17] A. Alavi, J. Kohanoff, M. Parrinello and D. Frenkel, Phys. Rev. Lett. 73, 2599 (1994).

[18] An elegant expression for the partition function of the total system (electrons + ions) can be obtained if one uses the alternate expression $\operatorname{det}\left(\mathbf{M}_{l}^{\dagger} \mathbf{M}_{l}\right)^{\frac{1}{2}}=\frac{\int \mathcal{D}\left[\boldsymbol{\Phi}_{l}\right] e^{-\mathbf{\Phi}_{l}^{\dagger}\left(\mathbf{M}_{l}^{\dagger} \mathbf{M}_{l}\right)^{-1} \mathbf{\Phi}_{l}}}{\int \mathcal{D}\left[\mathbf{\Phi}_{l}\right] e^{-\boldsymbol{\Phi}_{l}^{\dagger} \boldsymbol{\Phi}_{l}}}$, leading to $Z \propto \int d R_{I} e^{-\beta V_{r}} \int \prod_{l=1}^{P} \mathcal{D}\left[\mathbf{\Phi}_{l}\right] e^{-\sum_{l=1}^{P} \mathbf{\Phi}_{l}^{\dagger}\left(\mathbf{M}_{l}^{\dagger} \mathbf{M}_{l}\right)^{-1} \mathbf{\Phi}_{l}}$. Unfortunately this led to an algorithm which was less 
efficient than what is described here. This will be discussed in detail elsewhere.

[19] W. H. Press, S. A. Teukolsky, W. T. Vetterling and B. P. Flannery, Numerical Recipes in Fortran 7\%, (Cambridge University Press, Cambridge, 1992).

[20] D. M. Ceperley and M. Dewing, J. Chem. Phys. 110, 9812 (1999).
[21] L. Goodwin, A. J. Skinner and D. J. Pettifor, Europhys. Lett. 9, 701 (1989).

[22] A. Ricci and G. Ciccotti, Mol. Phys. 101, 1927, 2003.

[23] D. Shellman, J. P. Lewis, K. R. Glaesemann, K. Sikorski, and G. A. Voth, J. Comp. Phys. 188, 1 (2003). 